\title{
ON SOME ASYMPTOTIC FORMULAS IN THE THEORY OF PARTITIONS
}

\author{
PAUL ERDÖS
}

Let $p(n)$ denote the number of unrestricted partitions of $n . p_{k}(n)$ denotes the number of partitions of $n$ into precisely $k$ summands, or what is the same into partitions whose largest summand is $k$. Auluck, Chowla and Gupta ${ }^{1}$ announced the following conjecture:

For $n$ fixed let $p_{k_{0}}(n)$ be the greatest $p_{k}(n)$; that is, $p_{k_{0}}(n) \geqq p_{k}(n)$. Then

$$
k_{0} \sim c^{-1} n^{1 / 2} \log n, \quad c=\pi(2 / 3)^{1 / 2} .
$$

They prove that

$$
n^{1 / 2}<k_{0}<(1+\delta) c^{-1} n^{1 / 2} \log n
$$

for every $\delta>0$ if $n$ is sufficiently large.

In the present note we shall prove (1). In fact we shall prove that

$$
k_{0}=c^{-1} n^{1 / 2} \log n+a n^{1 / 2}+o\left(n^{1 / 2}\right) \quad \text { where } \quad c / 2=e^{-c a / 2} .
$$

They also conjectured that for $k_{1}<k_{2} \leqq k_{0}, p_{k_{1}}(n) \leqq p_{k_{2}}(n)$ and for $k_{0}<k_{1}<k_{2}, p_{k_{1}}(n)<p_{k_{2}}(n)$. They verify this conjecture for $n \leqq 32$. Recently Todd ${ }^{2}$ published a table of all the $p_{k}(n)$ for $n \leqq 100$, and it is easy to verify the conjecture for $n \leqq 100$. I am unable to prove or disprove this conjecture. They also remark that $p_{k_{0}}(n)$ differs from $c^{-1} n^{1 / 2} \log n$ by less than 1 for $n \leqq 32$; (2) shows that for large $n$ the difference tends to infinity.

Lehner and $\mathrm{I}^{3}$ proved that if we denote

$$
P_{k}(n)=\sum_{r \leqq k} p_{r}(n)
$$

then for $k=c^{-1} n^{1 / 2} \log n+\lambda n^{1 / 2}$ we have the asymptotic formula

$$
P_{k}(n) / p(n)=(1+o(1)) \exp \left(-(2 / c) e^{-c x / 2}\right) .
$$

In proving (2) we shall use (3) a great deal, we shall also use the well known asymptotic formula

$$
p(n)=(1+o(1))\left(1 / 4 \cdot 3^{1 / 2} n\right) \exp \left(c n^{1 / 2}\right) .
$$

Received by the editors March 12, 1945.

1 J. Indian Math. Soc. vol. 6 (1942) pp. 105-112.

2 Proc. London Math. Soc. vol. 48 (1944) pp. 229-242.

'Duke Math. J. vol. 8 (1941) pp. 335-345. 
Let $f(n)$ tend to infinity arbitrarily slowly; we easily obtain from (3) that for $k_{1}=\left[c^{-1} n^{1 / 2} \log n+f(n) n^{1 / 2}\right], k_{2}=\left[c^{-1} n^{1 / 2} \log n-f(n) n^{1 / 2}\right]$,

$$
(1 / p(n))\left(P_{k_{1}}(n)-P_{k_{2}}(n)\right) \rightarrow 1 \text { as } n \rightarrow \infty \text {. }
$$

We immediately obtain from (4) and (5) that for some $k_{2}<k_{3}<k_{1}$

$$
p_{k_{8}}(n)>c_{1} p(n) / n^{1 / 2}>\left(c_{2} / n^{3 / 2}\right) \exp \left(c n^{1 / 2}\right) \text {. }
$$

$c_{1}, c_{2}, \cdots$ denote absolute constants. Thus

$$
p_{k_{0}}(n) \geqq p_{k_{3}}(n)>\left(c_{2} / n^{3 / 2}\right) \exp \left(c n^{1 / 2}\right) .
$$

Now we show that for sufficiently large $c_{3}$

$$
k_{0}<c^{-1} n^{1 / 2} \log n+c_{3} n^{1 / 2} \text {. }
$$

Let $k_{4} \geqq c^{-1} n^{1 / 2} \log n+c_{3} n^{1 / 2}$. It clearly follows from the definition of $p_{k}(n)$ and $P_{k}(n)$ that $p_{k_{4}}(n)=P_{k_{4}}\left(n-k_{4}\right)<p\left(n-k_{4}\right)$. Thus from (4)

$$
\begin{aligned}
p_{h_{4}}(n) & <\left(c_{4} / n\right) \exp \left(c\left(n-k_{4}\right)^{1 / 2}\right)<\left(c_{4} / n\right) \exp c\left(n^{1 / 2}-k_{4} / 2 n^{1 / 2}\right) \\
& <\left(c_{4} / n\right) \exp \left(c\left(n^{1 / 2}-\log n / 2-c_{3} / 2\right)\right) \\
& <\left(c_{2} / n^{3 / 2}\right) \exp \left(c n^{1 / 2}\right)<p_{k_{0}}(n)
\end{aligned}
$$

for sufficiently large $c_{3}$, and this proves (8).

Next we prove that for sufficiently large $c_{s}$

$$
k_{0}>c^{-1} n^{1 / 2} \log n-c_{5} n^{1 / 2} .
$$

Suppose (9) does not hold. We obtain from (7) that for some $k_{0}<c^{-1} n^{1 / 2} \log n-c_{5} n^{1 / 2}$

$$
p_{k_{0}}(n)>\left(c_{2} / n^{3 / 2}\right) \exp \left(c n^{1 / 2}\right) .
$$

We shall show that (10) leads to a contradiction. First we show that

$$
p_{k}(n) \leqq p_{k+i}(n+j) \quad \text { for } j \geqq i \text {. }
$$

We have

$$
p_{k}(n) \leqq p_{k+i}(n+i) \leqq p_{k+i}(n+j) .
$$

The first inequality of (12) we obtain by mapping the partition $a_{1}+\cdots+k$ of $p_{k}(n)$ into $a_{1}+\cdots+(k+i)$ which belongs to $p_{k+i}(n+i)$, the second part we obtain by adding $j-i 1$ 's to every partition of $p_{k+i}(n+i)$; this proves (11).

Put $\left[n^{1 / 2}\right]=b$; we have from (10) and (11) for $0 \leqq i \leqq b$

Thus

$$
\begin{aligned}
p_{k_{0}+i}(n+b) & \geqq p_{k_{0}}(n)>\left(c_{2} / n^{3 / 2}\right) \exp \left(c n^{1 / 2}\right) \\
& >\left(c_{6} / n^{3 / 2}\right) \exp \left(c(n+b)^{1 / 2}\right) .
\end{aligned}
$$




$$
\sum_{i=0}^{b} p_{k_{0}+i}(n+b)>\left(c_{6} / n\right) \exp \left(c(n+b)^{1 / 2}\right) .
$$

Now we obtain from (5) that for every $\epsilon$ and sufficiently large $c_{5}$ and $n$

$$
\sum_{k>k_{0}+b} p_{k}(n+b)>(1-\epsilon) p(n+b) .
$$

The proof of (14) follows immediately from the fact that $k_{0}+b$ $\left\langle c^{-1} n^{1 / 2} \log n-\left(c_{5}-1\right) n^{1 / 2}\right.$, thus (5) can be applied. From (13) and (14) we have

Thus

$$
\begin{aligned}
p(n+b) & >\sum_{i=0}^{b} p_{k_{0}+i}(n+b)+\sum_{k>k_{0}+b} p_{k}(n+b) \\
& >(1-\epsilon) p(n+b)+\left(c_{6 / n}\right) \exp \left(c(n+b)^{1 / 2}\right) .
\end{aligned}
$$

$$
\epsilon p(n+b)>\left(c_{b / n}\right) \exp \left(c(n+b)^{1 / 2}\right),
$$

which contradicts (4); this proves (9).

We now know from (8) and (9) that $k_{0}$ has to satisfy

$$
c^{-1} n^{1 / 2} \log n-c_{5} n^{1 / 2}<k_{0}<c^{-1} n^{1 / 2} \log n+c_{3} n^{1 / 2} \text {. }
$$

Put

$$
k_{0}=c^{-1} n^{1 / 2} \log n+x n^{1 / 2} .
$$

We obtain from (3) and (4) that

$$
\begin{aligned}
p_{k_{0}}(n) & =P_{k_{0}}\left(n-k_{0}\right) \\
& =(1+o(1)) p(n) n^{-1 / 2} \exp (-c x / 2-(2 / c) \exp (-c x / 2)) .^{4}
\end{aligned}
$$

The right side is maximal if $c / 2=\exp (-c x / 2)$, which completes the proof of (2).

We immediately obtain from (2) and (15) that

$$
\lim p_{k_{0}}(n) n^{1 / 2} / p(n)=\exp (-c a / 2-(2 / c) \exp (-a x / 2)) .
$$

It would be easy to sharpen the error term $o\left(n^{1 / 2}\right)$ in (2) by getting an error term in (3), but it seems very hard to get a sufficiently good inequality to prove the conjecture of Auluck, Chowla and Gupta.

Denote by $Q(n)$ the number of partitions of $n$ into unequal parts. $Q_{k}(n)$ denotes the number of partitions of $n$ into precisely $k$ unequal parts. Define $k_{0}$ by

$$
Q_{k_{0}}(n) \geqq Q_{k}(n) \text {. }
$$

- This formula is due to Auluck, Chowla and Gupta (ibid). 
It has been conjectured that for $k_{1}<k_{2} \leqq k_{0}, Q_{k_{1}}(n)<Q_{k_{2}}(n)$ and for $k_{0}<k_{1}<k_{2}, Q_{k_{1}}(n) \geqq Q_{k_{2}}(n)$. This conjecture we can not decide. But by using Theorem 3.3 of our paper with Lehner we can show that

$$
k_{0}=2 \log 2 n^{1 / 2} / \pi(1 / 3)^{1 / 2}+d n^{1 / 4}+o\left(n^{1 / 4}\right)
$$

for a certain constant $d$. Also

$\lim n^{1 / 4} Q_{k_{0}}(n) / Q(n) \rightarrow e, \quad$ for a certain constant $e$.

We do not discuss the proofs. They are similar but slightly more complicated than the proof of (2).

It would be interesting to get an asymptotic formula for $p_{k}(n)$ and $Q_{k}(n)$. Perhaps the first step would be to get an asymptotic formula for $\log p_{k}(n)$. It is easy to see that for $k=o\left(n^{1 / 2}\right)$

$$
\log p_{k}(n)=o\left(n^{1 / 2}\right)
$$

and if $k / n^{1 / 2} \rightarrow \infty$

$$
\log p_{k}(n) / \log p(n) \rightarrow 1 .
$$

The proofs can be obtained easily by simple Tauberian theorems.

UNIVERSITY OF MICHIGAN 